GESTÃO DA INFORMAÇÃO 


\title{
BLOCKCHAIN: PERFIL DAS PESQUISAS DIVULGADAS EM PERIÓDICOS ACADÊMICOS
}

\author{
BLOCKCHAIN: PROFILE OF RESEARCH PUBLISHED \\ IN ACADEMIC JOURNALS
}

Fernanda da Silva Momo

Universidade Federal do Rio Grande do Sul

Ariel Behr

Data de submissão: 28 fev. 20 | 8 . Data de aprovação:

12 nov. 2018. Sistema de avaliação: Double blind review.

Universidade FUMEC / FACE. Prof. Dr. Henrique Cordeiro

Martins. Prof. Dr. Cid Gonçalves Filho.

Universidade Federal do Rio Grande do Sul

RESUMO

A Blockchain é uma temática facilmente constatado em revistas de negócios e portais de tecnologia e destaca como uma tecnologia relevante e disruptiva para os negócios. Em seu conceito, destaca-se questões como descentralização, segurança (criptografia), confiança, automatização e publicidade das informações. Assim, no intuido de melhor situar o assunto Blockchain no contexto acadêmico, o presente estudo almeja destacar o perfil das pesquisas divulgadas em periódicos científicos sobre Blockchain, sitematizando assim as abordagens dadas a esse tema na pesquisa científica e contribuindo para a formação de agendas de pesquisa sobre o assunto. Para isso, optou-se por um estudo quantitativo, por meio de uma revisão sistemática de literatura sobre o tema Blockchain, publicados até abril de 2017. Foram estabelecidas estratégias de buscas que resultaram em uma base de 28 artigos, analisados com abordagem bibliométrica e com análise de conteúdo, definindo categorias por meio da contagem frequencial. Como resultados, destaca-se que há uma pluralidade de abordagens dada a essa temática, tendo em vista a área de conhecimento dos periódicos em que foram publicados. Além disso, esse é um tema que já possui espaço para discussão de sua aplicabilidade e pesquisas em diversos países, sendo possível destacar países como Estados Unidos, Países Baixos e Reino Unido.

\section{PALAVRAS-CHAVE}

Blockchain. Bibliometria. Periódicos Acadêmicos. 


\section{ABSTRACT}

Blockchain is a subject easily found in business magazines and technology portals and stands out as a relevant and disruptive technology for business. In its concept, it's worth mentioning topics like decentralization, security (encryption), confidence, automation and publicity of the information. Thus, in order to better situate the Blockchain subject in the academic context, this study aims to highlight the profile of published studies in scientific journals on Blockchain, thereby systematizing the approaches to this theme in scientific research and contributing to the formation of research agendas about the subject. In order to that, a quantitative study was carried out, through a systematic review of the literature on the Blockchain theme published until April 2017. Search strategies has been established and resulted in a base of 28 articles, analyzed with a bibliometric approach and with content analysis, defining categories by frequency counting. As results, it is highlighted that there are a plurality of approaches on this subject, considering the area of knowledge of the periodicals in which they were published. In addition, this is a topic that already has room for discussion of its applicability and research in various countries, being possible to point out various countries such as the United States, the Netherlands and the United Kingdom.

\section{KEYWORDS}

Blockchain. Bibliometria. Academic Newspapers.

\section{INTRODUÇÃO}

Fernando é o Administrador de uma grande empresa de consultoria e a pouco tempo ganhou um livro em que, na sua descrição, lhe chamou atenção a seguinte frase: 'A tecnologia que provavelmente terá o maior impacto sobre o futuro da economia mundial chegou, e não é o carro autônomo, a energia solar ou a inteligência artificial. Chama-se Blockchain.' (TAPSCOTT;TAPSCOTT, 20I6, p. I). Além disso, já faz tempo que Fernando observa títulos de notícias como 'Porque Blockchain irá mudar as organizações?'; 'Blockchain: a internet dos negócios'; 'Revolução do Blockchain vai transformar a maneira como os negócios são feitos'; 'A tecnologia que [...] tem o potencial de revolucionar a economia mun- dial'; 'Blockchain desafia grandes bancos'; 'Porque Blockchain pode ser sua próxima cadeia de suprimentos?' (VALDES; FURLONGER, 20I6; TAPSCOTT; KIRKLAND, 2016; STELER; CERQUEIRA, 20I7; NERY, 2017; MCKENDRICK, 20I7; TAPSCOTT; TAPSCOTT, 20I7).

Como o caso inicial ilustra, o interesse pelo tema da Blockchain é facilmente constatado em revistas de negócios e portais de tecnologia. Muitas das notícias enfocam a relevância dessa tecnologia para os negócios e seu potencial disruptivo para a forma de se fazer negócios. E de forma sumária, pode-se dizer que Blockchain é um banco de dados distribuído e compartilhado, tendo como base a questão da criptografia para garantir a autenticidade da informa- 
ção (WRIGHT E DE FILIPE, 20I5). Cabe ressalvar também que a Blockchain é uma tecnologia que surgiu em função do Bitcoin (uma moeda digital), entretanto, não tem como aplicação exclusiva o Bitcoin. Em seu conceito destacam-se características como a descentralização, segurança (criptografia), confiança, automatização e publicidade das informações.

$\mathrm{E}$ a fim de melhor situar o assunto Blockchain no contexto acadêmico, o presente estudo almeja responder a seguinte questão de pesquisa: Qual o perfil das pesquisas divulgadas em periódicos científicos sobre Blockchain? Assim, o objetivo proposto que irá conduzir a pesquisa é sistematizar as abordagens dadas para a temática de Blockchain na pesquisa científica, e assim, tem-se como resultado esperado, contribuir para a formação de agendas de pesquisa sobre $o$ assunto.

No que tange aos procedimentos utilizados para operacionalizar esse objetivo, optou-se por um estudo quantitativo, por meio de uma revisão sistemática de literatura sobre o tema Blockchain, em que a coleta dos textos foi realizada abril de 2017 . Foram estabelecidas estratégias de buscas que resultaram em uma base de $\mathbf{2 8}$ artigos, analisados com abordagem bibliométrica e com análise de conteúdo (BARDIN, 20II), definindo categorias por meio da contagem frequencial.

O presente artigo estrutura-se trazendo a presente introdução e, em seguida, na seção 2, os conceitos relacionados à Blockchain. Na seção 3 são apresentados os procedimentos metodológicos adotados na pesquisa e, na seção 4, são apresentados os resultados e respectivas análises. $\mathrm{E}$, por fim, apresentam-se na seção 5 as considerações finais.

\section{BLOCKCHAIN}

Blockchain surgiu no intuito de desenvolver uma tecnologia que permitisse, nas transações financeiras, a exclusão do agente intermediário. Nesse sentido, Nakamoto (2008, p. I), tendo em vista que "comércio na Internet passou a depender quase exclusivamente de instituições financeiras que servem como terceiros confiáveis para processar pagamentos eletrônicos", destacou como necessário o desenvolvimento de um "sistema de pagamento eletrônico baseado em prova criptográfica em vez de confiança" de forma a permitir "quaisquer duas partes dispostas a negociar diretamente uns com os outros sem a necessidade de um terceiro de confiança".

Nesse contexto, a partir da publicação de um artigo, Nakamoto propôs uma solução para o problema da dupla despesa quando há um intermediário envolvido na transação a partir de um método peerto-peer distribuído que validou o Bitcoin como moeda virtual e caracterizou-se como a tecnologia Blockchain (NAKAMOTO, 2008; SWAN, 20I5; YERMACK, 20I7). Assim, tem-se que após "7 anos de uso bem-sucedido com Bitcoin, a Blockchain tornou-se reconhecida como uma alternativa aos livros-razão da propriedade baseados na contabilidade clássica das partidas dobradas" (YERMACK, 2017, p.8). Portanto, é notório que o Bitcoin "não é uma característica de definição do Blockchain, mas sim uma mera aplicação da mesma" (PILSINGYON, 20I6, p. I0).

Dessa forma, o uso da Blockchain leva a reduzir de forma potencial o papel dos intermediários, um dos mais importantes atores regulatórios, na sociedade uma vez que descentraliza a forma como é feito o armazenamento de dados e gerenciamen- 
to de informações (WRIGHT; DE FILIPPI, 2015). Assim, pode-se vislumbrar a Blockchain com uma "consequência visível (embora intangível) das ações tomadas pelos usuários de uma rede" (PILKINGTON, 2016, p.10).

Além dessa questão do intermediário, destaca-se também a relevância social da Blockchain para a inclusão social no mundo em desenvolvimento tendo em vista que muitas pessoas não participam do mercado online por não terem acesso a contas nos intermediários atuais do mercado (PILKINGTON, 20I6; TAPSCOTT; TAPSCOTT, 2017). Sendo assim, a Blockchain emergiu como uma "inovação disruptiva com uma ampla gama de aplicações, potencialmente capaz de redesenhar nossas interações nos negócios, na política e na sociedade em geral" (ATZORI, 20I5, p. I).

Em relação ao conceito do Blockchain, destaca-se a definição dos autores Wright e De Filipe (20I5, p.2) que descrevem a Blockchain como um "banco de dados distribuído, compartilhado e criptografado que serve como um repositório público irreversível e incorruptível de informações". Além disso, esses autores complementam esse definição ressaltando o fato de que, "pela primeira vez, é possível que pessoas não relacionadas cheguem a um consenso sobre a ocorrência de uma determinada transação ou evento sem a necessidade de uma autoridade de controle" reforçando dessa forma todo o contexto e características destacadas até esse momento em relação a Blockchain.

Tendo em vista o que foi apresentado anteriormente em relação ao tema Blockchain, apresentam-se os sete princípios básicos para auxiliar no entendimento da Blockchain, uma vez que esses princípios estão relacionados às características relativas a Blockchain. Esses princípios são: Integridade na rede (Networked Integrity); Potência distribuída (Distributed Power); Valor como incentivo (Value as Incentive); Segurança (Security); Privacidade (Privacy); Direitos preservados (Rights Preserved); Inclusão (Inclusion). Nesse sentido, esses sete princípios podem servir como guia para "projetar a próxima geração de organizações inovadoras e de alto desempenho" (TAPSCOTT;TAPSCOTT, 2016 , p. 52).

Em uma outra perspectiva, Swan (2015, p. II) apresenta três categorias da Blockchain que destacam os diferentes tipos de atividades existentes e potenciais na revolução Blockchain, tendo em vista seu caráter disruptivo. Nas palavras do autor, as três categorias destacadas foram: Blockchain I.0, 2.0 e 3.0.

Blockchain 1.0 é moeda, a implantação de criptocorrências em aplicações relacionadas ao dinheiro, como transferência de moeda, remessa e sistemas de pagamento digital. Blockchain 2.0 é contratos, toda a ardósia de aplicações econômicas, de mercado e financeiras usando a cadeia de bloqueio que são mais extensas do que simples transações em dinheiro: ações, títulos, futuros, empréstimos, hipotecas, títulos, propriedade inteligente e contratos inteligentes. Blockchain 3.0 é Blockchain aplicações além de moeda, finanças e mercados, particularmente nas áreas de governo, saúde, ciência, alfabetização, cultura e arte. (SWAN, 20I5, p.II) 
Nessa categorização realizada pela Swan (20I5) é implícita a abrangência de usos dessa tecnologia. Assim, a Blockchain não está restrita a moedas descentralizadas e envolve também a criação de "contratos digitais auto executáveis (contratos inteligentes) e ativos inteligentes que podem ser controlados pela Internet (propriedade inteligente)" além de possibilitar o "desenvolvimento de novos sistemas de governança com uma tomada de decisão mais democrática ou participativa e organizações descentralizadas (autônomas) que podem operar através de uma rede de computadores sem qualquer intervenção humana" (WRIGHT; DE FILIPPI, 20I5, p. I, tradução nossa).

Outras aplicações da Blockchain que podem ser destacadas são o uso dessa tecnologia para registrar propriedades de ativos de diversas naturezas (ações, imóveis, automóveis, bolsas de luxo, obra de arte), e até a utilização para registros públicos como "títulos imobiliários, certidões de nascimento, carteiras de motorista e graus universitários" (YERMACK, 20I7, p. 8). Sendo assim, compreendendo as características inerentes a essa tecnologia e algumas de suas aplicações, é possível vislumbrar o porquê da Blockchain estar sendo considerada como a internet dos negócios, ao passo que essa descentralização, conforme Wright e De Filippi (20I5), pode afetar não apenas a aplicação das leis ou sua implementação como também os governos, organizações e a sociedade em geral em seu modo de operar.

\section{MÉTODO}

Os procedimentos metodológicos adotados na pesquisa que origina $\circ$ presente artigo podem ser enquadrados, quanto a sua natureza, como quantitativos, uma vez que norteiam-se pela objetividade, descrevendo as causas de um fenômeno por meio da linguagem matemática. Quanto aos objetivos, verifica-se que a pesquisa em questão é descritiva, pois descreve as características de uma amostra, utilizando técnicas padronizadas de coleta de dados. E quanto aos procedimentos técnicos, pode ser entendida enquanto uma pesquisa bibliográfica, tendo como dados para suporte às afirmações a serem feitas uma base de material já elaborado, constituída de artigos científicos (GIL, 2008).

As unidades de análise da pesquisa em questão foram 28 artigos publicados em periódicos científicos. Para coleta, ocorrida em abril de 2017, foram utilizadas as bases de dados EBSCOhost, Springer, Science Direct, Scopus eWeb of Science. Em cada uma dessas bases foram coletados todos os artigos que possuíam em seu título o termo Blockchain. Além disso, para essa coleta, era necessário que os artigos tivessem seu título em inglês, espanhol ou português. Assim, na Figura I, explicita-se mais detaIhadamente esses procedimentos, visando tornar este um processo transparente, explícito e reprodutível (MUÑOS, 2009).

Dessa forma, conforme Figura I, a busca primária realizada nas bases de dados retornou 80 artigos, dos quais 27 foram excluídos por não estarem publicados em periódicos acadêmicos, ou por não terem títulos em inglês, português ou espanhol. Dos 53 artigos restantes, identificou-se que 26 apareceram repetidamente nas diferentes bases de dados pesquisadas; assim, retiraram-se da base de dados as redundâncias de forma a identificar que esses 26 artigos se referem a 16 estudos. Portanto, restaram 37 artigos que tiveram seus arquivos 
analisados de maneira breve para verificar se fariam parte da amostra de análise e, portanto, estavam em acordo com as características definidas para este estudo, ou seja, se eram artigos científicos, publicados em periódicos acadêmicos, com o termo 'Blockchain' em seu título e escritos em inglês, espanhol ou português.

Nesta análise final, identificou-se que 9 artigos não estavam nos parâmetros definidos neste estudo e, por isso, foram excluídos. Em relação aos motivos das exclusões tem-se que 5 artigos não estavam escritos nos idiomas definidos, ou seja, houve um artigo em alemão e 4 em chinês que foram excluídos da amostra.Além disso, foi excluído um capítulo de livro, I editorial, I artigo que era um 'Method Research' e, por fim, excluiu-se um estudo que foi retraído pelos autores, ou seja, foi retirado na revista depois de constatações de fragilidades no método aplicado pelos autores em uma revisão pós-publicação.
Ao final desses procedimentos restaram para a análise 28 artigos que foram tabulados em planilha eletrônica com as informações de 'Ano de publicação', 'Base de dados de origem', 'Periódico', 'Autores', 'Título', 'Palavras-chave', 'Resumo', 'Método' e 'Resultados, conforme destacado na Figura I.Além disso, salvou-se em uma pasta o arquivo completo dos textos desses artigos, com exceção de um deles ao qual não foi possível conseguir acesso. No Apêndice $A$ estão apresentados os 28 artigos selecionados para análise.

A análise dos dados se deu de duas formas. A primeira utilizando procedimentos bibliométricos inspirados na Lei de Lotka (1926) ou Lei do Quadrado Inverso, que afirma que é um pequeno quantitativo de pesquisadores produz a maior parte dos artigos de uma área de conhecimento; e também inspirados na Lei Braford ou Lei da Dispersão (ROUSSEAU; ROUSSEAU, 2000) que suporta a afirmação de que um

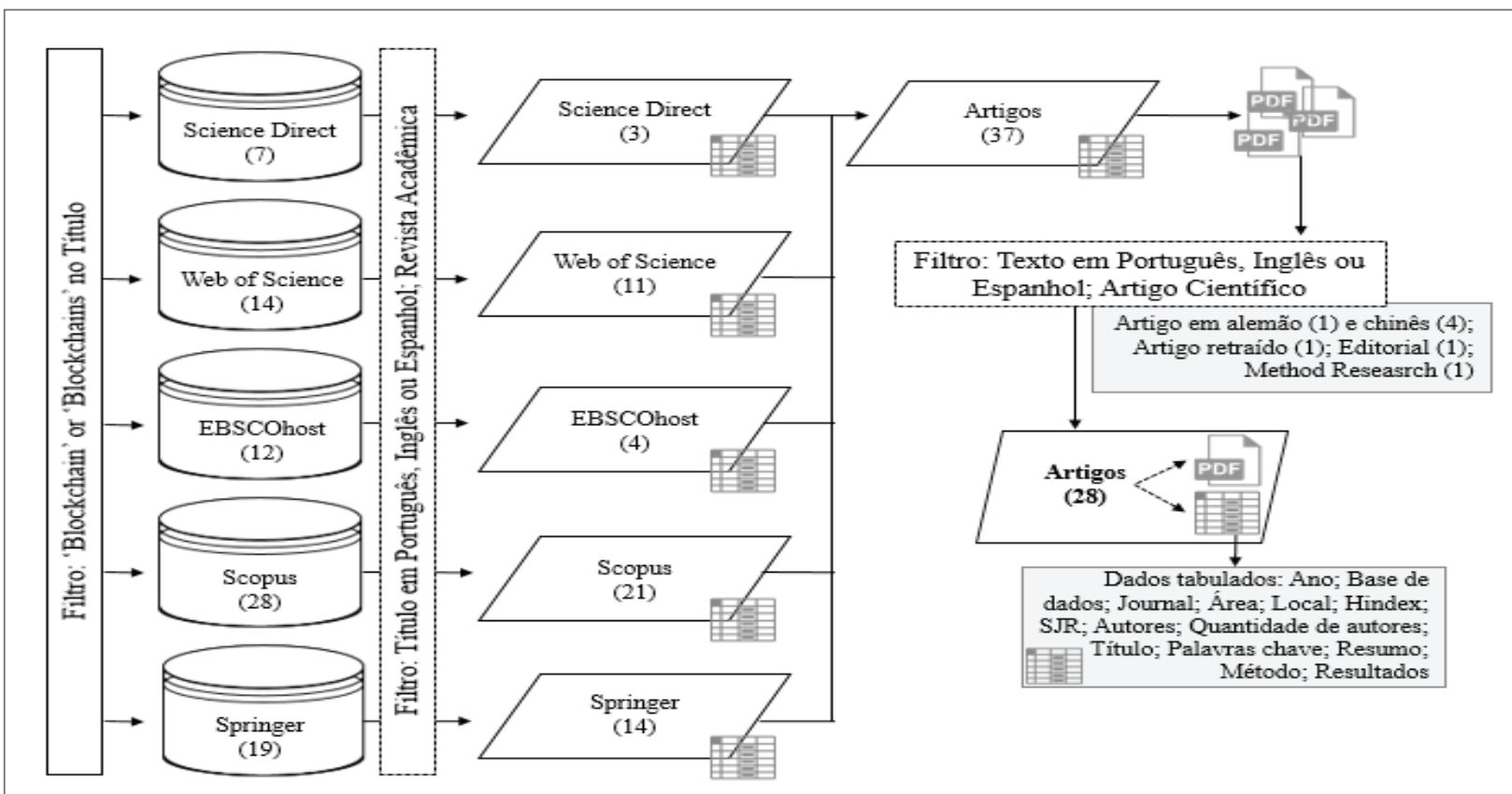

FIGURA 1 - Procedimentos de Coleta e Análise dos dados

Fonte: elaborado pelos autores (2019) 
pequeno grupo de periódicos científicos atende com maior profundidade certo assunto, pretendendo estimar o grau de relevância de periódicos que atuam em áreas do conhecimento. Assim, busca-se na presente pesquisa analisar a estrutura de autoria dos artigos relacionados ao tema em questão (Blockchain), identificar a quantidade de artigos publicados em revistas acadêmicas ao longo dos anos, os periódicos que possuem maior concentração de publicações sobre esse tema, a origem desses periódicos e a base de dados onde seus artigos foram encontrados.

A segunda forma de análise dos dados foi a análise de conteúdo (BARDIN, 20I I) empregando a categorização por meio da contagem frequencial, e interpretando $\circ$ significado dessas palavras quando associadas em conjuntos. Utilizou-se nesta etapa ○ software $\mathrm{NVivo} \AA$, versão II, a fim de operacionalizar a contagem frequencial e a associação de palavras em conjuntos. Nesta etapa, optou-se inicialmente por apresentar um panorama inicial em relação a todo conjunto de artigos selecionados e, posteriormente, um aprofundamento nos artigos relacionados a área de conhecimento de Ciências Sociais Aplicadas. Para essas análises utilizou-se dos documentos completos e a planilha elaborada. $\mathrm{Na}$ seção que segue são apresentadas as análises dos resultados da pesquisa.

\section{ANÁLISE DOS RESULTADOS}

No que tange a análise dos 28 artigos selecionados todos estão escritos na língua inglesa e a maioria deles, ou seja, 20 artigos (7I,42\%) foi publicado no ano de 2016. Entretanto, destaca-se que no ano de 2017 já foram publicados 7 artigos sobre o tema Blockchain e ainda há publicações que devem ser realizadas até o final do ano. Outra questão a ser observada é que a base de dados com mais artigos disponibilizados sobre este tema de forma exclusiva, ou seja, artigos que apareceram apenas em uma base de dados foi a Springer com II artigos. Em termos absolutos foi também essa base que apresentou uma maior quantidade de artigos como resultado da busca inicial. A Tabela I apresenta essas informações de forma e especificar a quantidade de publicações em cada base de dados em cada ano.

No que se refere aos periódicos em que foram publicados estes artigos, destaca-se que não houve nesse período uma grande concentração de publicações em um mesmo periódico. Sendo assim, dos 28 journals

TABELA 1 - Publicações por base de dados em cada ano

\begin{tabular}{l|c|c|c|c|c|c|c}
\hline Base de dados & $\mathbf{2 0 1 5}$ & $\begin{array}{c}\text { Artigos } \\
\text { Repetidos }\end{array}$ & $\mathbf{2 0 1 6}$ & $\begin{array}{c}\text { Artigos } \\
\text { Repetidos }\end{array}$ & $\mathbf{2 0 1 7}$ & $\begin{array}{c}\text { Artigos } \\
\text { Repetidos }\end{array}$ & $\begin{array}{c}\text { Total } \\
\text { Geral }\end{array}$ \\
\hline EBSCOhost & & 1 & 1 & & & 2 & 1 \\
\hline Science Direct & & 1 & 4 & 3 & & 4 & 4 \\
\hline Scopus & & & 9 & 2 & 2 & 1 & 11 \\
\hline Springer & & 1 & 1 & 4 & 1 & 3 & 2 \\
\hline Web of Science & & 1 & & 4 & & 4 & 9 \\
\hline $\begin{array}{l}\text { Total de artigos que apareceram em } \\
\text { mais de uma base de dados }\end{array}$ & $\mathbf{2 0 1 5}$ & 1 artigos & $\mathbf{2 0 1 6}$ & 20 artigos & $\mathbf{2 0 1 7}$ & 7 artigos & 28 \\
\hline Total Geral & & & & & 4 \\
\hline
\end{tabular}

Fonte: elaborado pelos autores (2019) 
que publicaram artigos sobre Blockchain, apenas 3 deles tiveram mais de um artigo publicado. Destaca-se também que essas publicações em um mesmo periódico ocorreram no ano de 2016 e podem ser visualizadas na Tabela 2 . Ainda em relação a essa análise, cabe destacar que o periódico Financial Innovation se destaca com 5 artigos sobre o tema Blockchain e isso está relacionado com o fato desse periódico ter lançado uma edição especial sobre esse tema.

Além disso, avaliou-se o país de origem desses periódicos e suas área de conhecimento (Apêndice $B$ ). Em relação ao país de origem, dos 21 periódicos, constatou-se que a maioria das publicações foram re- alizadas por journals dos Estados Unidos (33,33\%), Países Baixos (23,80\%) e Reino Unido (19,04\%). No que tange as áreas do conhecimento dos journals, a maioria é da área de Ciência da Computação (35,7l\%), seguido da área de Contabilidade, Finança e Economia $(21,43 \%)$ e Engenharia (7,14\%). Outros temas dos periódicos foram: Agricultura, Saúde, Artes, Negócios e Gestão, Ciência da Decisão, Computação Verde, Direito.A Tabela 3 demostra essas informações, informando a quantidade de artigos em cada área e o percentual em relação ao total de artigos (28).

No que se refere a autoria dos artigos, realizou-se inicialmente a análise dos autores dos artigos e identificou-se que nenhum

TABELA 2 - Periódicos com mais de uma publicação sobre Blockchain

\begin{tabular}{|c|c|c|c|c|c|c|}
\hline Periódico & \multicolumn{2}{|c|}{$\begin{array}{c}\text { Artigos publicados (todos } \\
\text { no ano 2016) }\end{array}$} & Área & Local & $H$ index & $\begin{array}{c}\text { SJR } \\
(2015)\end{array}$ \\
\hline $\begin{array}{l}\text { Peer-to-peer Networking and } \\
\text { Applications }\end{array}$ & 2 & \multirow{3}{*}{$\begin{array}{l}\stackrel{0}{-} \\
\frac{.}{\pi} \\
\frac{0}{0}\end{array}$} & Computer Science & United States & 10 & 0,395 \\
\hline Philosophy \& Technology & 3 & & Arts and Humanities & Netherlands & 8 & 0,311 \\
\hline Financial Innovation & 5 & & $\begin{array}{c}\text { Accounting, Finance \& } \\
\text { Economics }\end{array}$ & China & - & - \\
\hline
\end{tabular}

Fonte: elaborado pelos autores (2019)

TABELA 3 - Áreas de conhecimento dos periódicos e países

\begin{tabular}{l|c|c}
\hline \multicolumn{1}{c|}{ ÁREA } & QTD & \% \\
\hline Ciências Sociais e Aplicadas & $\mathbf{8}$ & $\mathbf{2 8 , 5 7 \%}$ \\
\hline Accounting, Finance \& Economics & 6 & $21,43 \%$ \\
\hline Business, Management and Accounting & 1 & $3,57 \%$ \\
\hline Social Sciences - Law & 1 & $3,57 \%$ \\
\hline Ciências Exatas e da Terra & $\mathbf{1 2}$ & $\mathbf{4 2 , 8 6 \%}$ \\
\hline Digital Society and Green Computing & 1 & $3,57 \%$ \\
\hline Internet technologies and the information society & 1 & $3,57 \%$ \\
\hline Computer Science & 10 & $35,71 \%$ \\
\hline Engenharias & $\mathbf{2}$ & $\mathbf{7 , 1 4 \%}$ \\
\hline Engineering & 2 & $7,14 \%$ \\
\hline Ciências da Saúde & $\mathbf{2}$ & $\mathbf{7 , 1 4 \%}$ \\
\hline Health Professions; Computer Science & 1 & $3,57 \%$ \\
\hline Medicine; Agricultural and Biological Sciences & 1 & $3,57 \%$ \\
\hline Linguística, Letras e Artes & $\mathbf{4}$ & $\mathbf{1 4 , 2 9 \%}$ \\
\hline Arts and Humanities & 4 & $14,29 \%$ \\
\hline
\end{tabular}

Fonte: elaborado pelos autores (2019) 
autor ou coautor publicou mais de um artigo nesse período. Esse resultado pode estar relacionado ao fato dessa temática de pesquisa ser recente e o ano mais antigo de publicação dos artigos analisados ter sido 20I5. Assim, tendo em vista esse resultado, optou-se por realizar a contagem da quantidade de autores nos artigos para identificar algum perfil em relação a autoria dos artigos. Com isso, verificou-se que a maioria dos artigos publicados possui dois, um e três autores respectivamente. Além disso, constatou-se que a quantidade máxima de autores em um mesmo artigo foi seis e ocorreu em uma publicação de 2017. A Tabela 4 a seguir ilustra bem essa análise.

Posteriormente a essas análises, avaliouse a partir da análise de frequência os conteúdos desses artigos de forma a identificar os temas pesquisados até o momento sobre Blockchain.Assim, será apresentada uma breve análise relativa as palavras e temas mais utilizados nessas partes do texto tendo em vista a frequência com que essas palavras aparecem no Título, Resumos, Texto completo. A Figura 2 apresenta as 20 palavras mais frequentes nos títulos dos artigos, excluídas as palavras Technology e Blockchain.

TABELA 4 - Quantidade de autores nos artigos

\begin{tabular}{c|c|c|c|c}
\hline Quantidade de autores & $\mathbf{2 0 1 5}$ & $\mathbf{2 0 1 6}$ & $\mathbf{2 0 1 7}$ & Total Geral \\
\hline 1 & 1 & 5 & 1 & 7 \\
\hline 2 & & 8 & 1 & 9 \\
\hline 3 & & 3 & 3 & 6 \\
\hline 4 & & 2 & 1 & 2 \\
\hline Total Geral & 5 & & 2 & 1 \\
\hline
\end{tabular}

Fonte: elaborado pelos autores (2019)

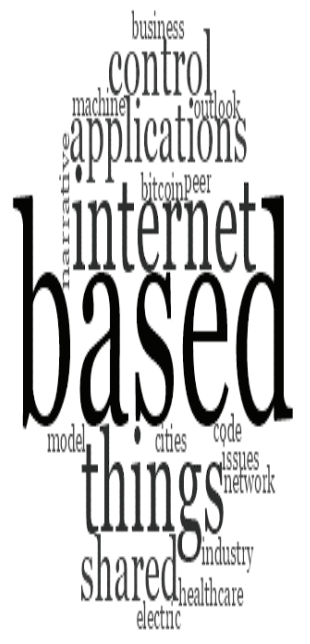

FIGURA 2 - Frequência das palavras dos Títulos dos artigos

Fonte: elaborado pelos autores (2019) 
Dentre as 20 palavras mais frequentes nos títulos dos artigos apresentados na Figura 2, destacam-se as que aparecem em no mínimo 3 títulos: 'Based'; 'Internet'; 'Things'; 'Applications'; 'Control'; 'Shared'. A palavra 'Based' é utilizada para se referir que o desenvolvimento do tema do artigo está baseado na tecnologia Blockchain; portanto, essa palavra pode ser vislumbrada, tendo em vista o contexto no qual ela é utilizada, como um sinônimo de Blockchain (SHARMA; MOON; PARK, 20I7; DENNIS; OWENSON, 2016; KRAFT, 2016; OUADDAH; ELKALAM; OUAHMAN, 2016; ZHANG, 20I6;SUN;YAN;ZHANG, 20I6). Destaca-se ainda que o uso dela é costumeiramente dado com um hífen ('Blockchain-based'), reforçando ainda mais essa relação explicitada.

No que se refere as palavras 'Internet' e 'Things', elas são utilizadas nos títulos dos artigos em conjunto como 'Internet of Things' (Internet das Coisas - loT). Assim, essas palavras estão relacionadas ao uso da Tecnologia Blockchain no contexto da IOT (HUCKLE; BHATTACHARYA;WHITE; BELOFF, 20 I 6; LEE; LEE, 20 I 7; OUADDAH; ELKALAM; OUAHMAN, 2016; ZHANG; WEN, 20I6). Em relação a palavra 'Applications' ela foi utilizada no sentido de pesquisar sobre aplicações da Blockchain no contexto da Economia Compartilhada; Bancos e em relação a Equidade (HUCKLE; BHATTACHARYA;WHITE; BELOFF, 20I6; ZHUZACH; ZHOU, 20I6; GUO; LIANG, 2016).

Já em relação a palavra 'Control', ela foi utilizada no sentido de abordar o tema de controle do risco de privacidade; dificuldade de controle das informações tendo em vista a característica de distribuição da informação e em relação ao estudo de uma nova estrutura de controle de acesso baseada nessa tecnologia em relação a loT (YUE;WANG; JIN; LI; JIANG, 20I 6; KRAFT, 2016; OUADDAH; ELKALAM; OUAHMAN, 20I6). Por fim, tem-se a palavra 'Shared' que foi utilizada quando abordado o tema da Economia Compartilhada; como característica das informações da Blockchain (informações compartilhadas) e em relação ao estudo de serviços compartilhados baseados nessa tecnologia (HUCKLE; BHATTACHARYA; WHITE; BELOFF, 2016; ZHANG, 20I6; SUN;YAN; ZHANG, 2016).

Assim, em termos gerais, as palavras mais frequentes destacadas remetem ao conceito de Blockchain ou aplicações que estão sendo estudadas para $\circ$ uso dessa tecnologia. Nesse sentido, reforça-se, como afirmado por alguns autores (YERMACK, 20I7; PILSINGYON, 20I6) que a tecnologia Blockchain que surgiu a serviço do bitcoin, já não se restringe mais a essa aplicação. As palavras também podem ser vislumbradas juntamente com a área de cada periódico o que reforça ainda mais a pluralidade de usos da Blockchain. A seguir apresenta-se o Quadro I que ilustra a análise de frequência dos títulos e resumos dos artigos analisados tendo em vista a área de conhecimento dos periódicos que foram publicados os artigos.

O Quadro I permite identificar que as pesquisas referentes a área de conhecimento de Ciências Sociais Aplicadas estão abordando o tema da Blockchain em perspectivas que envolvam as palavras 'Fraud', 'Based', 'Attack','Regulatory', 'Applications'. Nesse sentido, 'Fraud' (fraude) é utilizada juntamente com Blockchain para a discussão da eficácia dessa tecnologia em fraude objetiva e sua limitação na fraude subjetiva, especialmente a fraude de rating (classificação) no contexto do sistema de reputação 
QUADRO 1 - Frequência das palavras dos Títulos dos artigos e Resumos dos artigos por áreas

\begin{tabular}{|c|c|c|}
\hline Áreas e Artigos & Área & $\begin{array}{c}\text { Palavras mais frequentes nos Títulos e } \\
\text { Resumos }\end{array}$ \\
\hline Accounting, Finance \& Economics & \multirow{11}{*}{$\begin{array}{l}\frac{0}{\pi} \\
\frac{\pi}{0} \\
\frac{\pi}{0} \\
\frac{0}{0} \\
\frac{0}{2} \\
\frac{.0}{\pi} \\
\frac{\pi}{0} \\
0 \\
0 \\
\frac{0}{0} \\
\frac{\pi}{0} \\
\frac{0}{0}\end{array}$} & \multirow{11}{*}{$\begin{array}{l}\text { Fraud (14); Based (11); Attack (9); Regulatory } \\
\text { (8); Applications (7) }\end{array}$} \\
\hline A maturity model for blockchain adoption & & \\
\hline $\begin{array}{l}\text { Analysis and outlook of applications of blockchain technology to } \\
\text { equity crowdfunding in China }\end{array}$ & & \\
\hline Blockchain application and outlook in the banking industry & & \\
\hline $\begin{array}{l}\text { Blockchain-based sharing services: What blockchain technology can } \\
\text { contribute to smart cities }\end{array}$ & & \\
\hline $\begin{array}{l}\text { Fraud detections for online businesses: a perspective from } \\
\text { blockchain technology }\end{array}$ & & \\
\hline Regulatory Issues in Blockchain Technology & & \\
\hline Business, Management and Accounting & & \\
\hline Trusting records: is Blockchain technology the answer? & & \\
\hline Social Sciences - Law & & \\
\hline Beyond Bitcoin: issues in regulating blockchain transactions & & \\
\hline Digital Society and Green Computing & \multirow{15}{*}{ 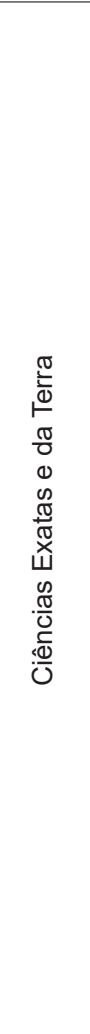 } & \multirow{15}{*}{$\begin{array}{l}\text { Internet (15); Firmware (14); Things (13); } \\
\text { Based (12); Code (12) }\end{array}$} \\
\hline $\begin{array}{l}\text { Rep on the Roll: A Peer to Peer Reputation System Based on a } \\
\text { Rolling Blockchain }\end{array}$ & & \\
\hline Internet technologies and the information society & & \\
\hline Socialism and the blockchain & & \\
\hline Computer Science & & \\
\hline Behavior pattern clustering in blockchain networks & & \\
\hline $\begin{array}{l}\text { Bitcoin blockchain dynamics: The selfish-mine strategy in the } \\
\text { presence of propagation delay }\end{array}$ & & \\
\hline Blockchain & & \\
\hline $\begin{array}{l}\text { Blockchain technology as a regulatory technology: From code is law } \\
\text { to law is code }\end{array}$ & & \\
\hline $\begin{array}{l}\text { Blockchain-based secure firmware update for embedded devices in } \\
\text { an Internet of Things environment }\end{array}$ & & \\
\hline $\begin{array}{l}\text { BlockVN: A Distributed Blockchain Based Vehicular Network } \\
\text { Architecture in Smart City }\end{array}$ & & \\
\hline Difficulty control for blockchainbased consensus systems & & \\
\hline $\begin{array}{l}\text { FairAccess: a new Blockchain-based access control framework for } \\
\text { the Internet of Things }\end{array}$ & & \\
\hline Internet of Things, Blockchain and Shared Economy Applications & & \\
\hline $\begin{array}{l}\text { The loT electric business model: Using blockchain technology for the } \\
\text { internet of things }\end{array}$ & & \\
\hline Engineering & \multirow{3}{*}{ 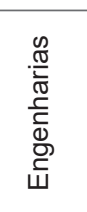 } & \multirow{3}{*}{$\begin{array}{l}\text { Information (6); Data (5); Method (5); Shared } \\
\text { (5); Electricity (4) } \\
\text { shared }\end{array}$} \\
\hline $\begin{array}{l}\text { Blockchain technology in the chemical industry: Machine-to-machine } \\
\text { electricity market }\end{array}$ & & \\
\hline $\begin{array}{l}\text { Walks trajectory tracking of shared information based on consortium } \\
\text { blockchain }\end{array}$ & & \\
\hline Health Professions; Computer Science & \multirow{4}{*}{$\begin{array}{l}0 \\
\frac{0}{0} \\
\frac{\pi}{\pi} \\
0 \\
\frac{\pi}{0} \\
\frac{0}{0} \\
\frac{\pi}{0} \\
\frac{\pi}{0}\end{array}$} & $\begin{array}{l}\text { Data (13); Healthcare (13); Patient (7); } \\
\text { Research (7); Privacy (5) }\end{array}$ \\
\hline $\begin{array}{l}\text { Healthcare Data Gateways: Found Healthcare Intelligence on } \\
\text { Blockchain with Novel Privacy Risk Control }\end{array}$ & & research \\
\hline Medicine; Agricultural and Biological Sciences & & \\
\hline $\begin{array}{l}\text { Where Is Current Research on Blockchain Technology?_A } \\
\text { Systematic Review. }\end{array}$ & & \\
\hline
\end{tabular}




\begin{tabular}{|l|c|}
\hline Arts and Humanities & Digital (15); Technologies (11); \\
\hline Digital Art as 'Monetised Graphics': Enforcing Intellectual Property on \\
the Blockchain
\end{tabular}

Fonte: elaborado pelos autores (2019)

para compras online. Em relação a palavra 'Based', ela foi utilizada como um complemento do termo Blockchain, ou para explicitar a teoria de base que se usaria na pesquisa. A palavra 'Attack' foi utilizada no sentido de enfatizar os objetivos (attack goals) e também para explicitar os tipos de ataques testados para verificar questões de eficácia da Blockchain em relação a 'Fraud'.

'Regulatory' aparece nos estudos analisados no contexto de verificar se a Blockchain resolve problemas relacionados a Compliance (conformidade) no contexto organizacional ajudando a diminuir o conflito de agência existente e, assim, proteger os direitos e os interesses dos pequenos investidores. Há também o uso dessa palavra no sentido de tentar aumentar a compreensão da comunidade dessa tecnologia e para informar os formuladores de políticas e os profissionais à medida que consideram diferentes esquemas regulatórios. Além dessas abordagens, destaca-se também que alguns autores propõem o estabelecimento de uma regulação em relação a Blockchain no contexto bancário, apesar da natureza sem permissão e autônoma dessa tecnologia. Seguindo essa abordagem apresentada, há também o uso de 'Regulatory' no sentido de examinar os principais desafios regulatórios que afetam a Blockchain na Europa e Estados Unidos.

$\mathrm{Na}$ área de Ciências Exatas e da Terra, destacou-se as palavras 'Internet', 'Firmware', 'Things', 'Based', 'Code'. 'Internet' foi utilizada no sentido de comportamento dos usuários da Internet; ambiente de Internet das coisas (loT) e ressaltando a loT como uma revolução inovadora pela internet. 'Firmware' foi utilizado no contexto de uma atualização de 'firmware' segura baseada em Blockchain na loT. Em relação a palavra 'Things' ressalta-se que ela está totalmente relacionada ao termo Internet das coisas (loT). A palavra 'Based', assim como ocorreu na análise dos títulos dos artigos, aparece como um complemento do termo Blockchain. Por fim, o termo 'Code' (código) é utilizado no contexto de regulação de forma que se apresenta, por exemplo, na questão de que com a tecnologia Blockchain e dos contratos inteligentes associados, o código está assumindo um papel ainda mais forte na regulação das interações das pessoas pela Internet.

Em reação a área de Engenharias, tem-se as palavras 'Information', 'Data', 'Method', 'Shared', 'Electricity' como mais frequentes. A palavra 'Information' é utilizada juntamente com a palavra 'Shared', de forma a destacar a característica da Blockchain de possuir as informações distribuídas. No que se refere a palavra 'Data', essa foi utilizada também juntamente a palavra 'Shared', contribuindo ainda mais para a exploração dessa característica de dados ou informações distribuídas da Blockchain. A outra aplicação dessa palavra ocorre juntamente com a palavra 'Block' destacando que as informações da Blockchain são ar- 
mazenadas em blocos. A palavra 'Method' é utilizada tanto para explicitar o método utilizado nos artigos, como também para mencionar métodos propostos que surgiram da realização da pesquisa. Por fim, em relação a palavra 'Electricity', ela é utilizada para a exploração de aplicações da Blockchain à $4^{\circ}$ Revolução Industrial, também chamada de Indústria 4.0.

No que se refere a área de Ciências da Saúde as palavras destacadas foram: 'Data', 'Healthcare', 'Patient', 'Research', 'Privacy'. A palavra 'Data' (Dados) se refere principalmente a dados de saúde, privacidade e segurança desses dados. Assim, pode-se contatar uma forte relação entre essa palavra ('Data') e 'Privacy (privacidade). As palavras 'Healthcare' e 'Patient' então relacionadas nos artigos como a área de estudo e o objeto/foco de estudo que, em grande parte dos artigos, está relacionada com o paciente. 'Research' (pesquisa) aparece no sentido de compreender os tópicos já abordados em pesquisas, áreas de pesquisas, auto referência da pesquisa e sugestões para futuras pesquisas.

Por fim, na área Linguística, Letras e Artes destacara-se as palavras 'Digital', 'Technologies', 'Images', 'Consciousness', 'Article'. A palavra 'Digital' foi utilizada juntamente com arte para destacar a forma de arte ao qual se estava enfocando a questão da propriedade intelectual. Outro uso dessa palavra ocorre juntamente com a palavra 'Technologies' ao destacar que as Tecnologias Digitais estão mudando as formas de criar e consumir narrativas. Por fim, utilizou-se também essa palavra no contexto de experiências digitais e para expressar o termo fenômenos digitais ao se referir ao bitcoin como um fenômeno digital ligado ao dinheiro e às finanças.
Em relação a palavra 'Technologies', essa foi utilizada juntamente com as palavras Blockchain, Cryptocurrency, Financial e Distributed Ledger. Esses termos se referem a característica da Blockchain (distributed ledger) ou até mesmo suas aplicações (cryptocurrency e financial). A palavra 'Images' (imagem) é utilizada no sentido de expressar a ideia de imagens em movimento e se referir a uma lente conceitual denominada imagem técnica de Vilém Flusser. No que tange a palavra 'Consciousness' (consciência), essa foi utilizada como uma perspectiva da teoria de Flusser. Já a palavra 'Article' (artigo) foi utilizada como uma auto referência, ou seja, para enfatizar, por exemplo, o que o artigo objetivava e iria abordar no decorrer do texto.

Sendo assim, a partir dessa análise é possível reforçar a questão da variedade de possibilidades do uso da Blockchain.Assim, ressalta-se que os estudos não estão apenas voltados para a área mais técnica de como a Blockchain deve estar estruturada, mas abordam-se também temas de regulação, e aplicações em diversas áreas. A tecnologia Blockchain não é limitada ao Bitcoin, sendo essa apenas uma aplicação dela, uma vez que nos 28 artigos publicados se observou uma diversidade de abordagens e uso dessa tecnologia em áreas de conhecimento de Ciências Sociais Aplicadas, Ciências Exatas e da Terra, Engenharias, Ciência da Saúde e Linguística, Letras e Artes. $O$ Quadro 2 traz um resumo do perfil dos trabalhos sobre Blockchain, tendo em vista suas áreas de conhecimento.

Tendo em vista explorar mais as pesquisas da área de conhecimento de Sociais Aplicadas, mais especificamente de 'Accounting, Finance \& Economics' e 'Business, Management and Accounting', apresenta- 


\section{QUADRO 2 - Perfil dos trabalhos publicados sobre Blockchain por áreas do conhecimento}

Discussões relacionadas a Blockchain nas diversas áreas do conhecimento:

\section{Ciências Sociais Aplicadas}

- eficácia dessa tecnologia em fraude.

- Blockchain como ferramenta para resolução de problemas relacionados a Compliance (conformidade) no contexto organizacional.

- dissiminação da compreensão dessa tecnologia pela comunidade do 'business'

- proposições de regulação e principais desafios regulatórios em relação a Blockchain

\section{Ciências Exatas e da Terra}

- uso da loT para pontencializar as aplicações de Blockchain

- desenvolvimento tecnológico da Blockchain (resolução de problemas e aprimoramento dessa tecnologia)

Engenharias

- aprimoramento da tecnologia, uso de dados

- exploração de aplicações da Blockchain à $4^{\circ}$ Revolução Industrial, também chamada de Indústria 4.0.

\section{Ciências da Saúde}

- possibilidade de aplicação da tecnologia blockchain para escriturar os dados de saúde em geral de pacientes

- discussão do uso da tecnologia com dados de saúde e a privacidade dos dados dos pacientes

\section{Linguística, Letras e Artes}

- possibilidade de escrituração e registro de propriedade intelectual

- destaque das Tecnologias Digitais como agentes que estão mudando as formas de criar e consumir narrativas

- direitos autorais

Fonte: elaborado pelos autores (2019)

se a seguir a Tabela 5 . Nessa tabela sumarizam-se os objetivos dos estudos dessas duas subáreas de Ciências Sociais Aplicadas e respectivos resultados.

A Tabela 5, ressalta, portanto, a partir dos resultados das pesquisas publicadas nas áreas 'Accounting, Finance \& Economics' e 'Business, Management and Accounting', um grande potencial de uso da Blockchain nos negócios e também algumas limitações. Em relação a esses sete artigos, cabe destacar o interesse em fomentar uma discussão em relação a regulamentação dessa tecnologia para um maior impacto e uso no mundo corporativo. Além disso, há a intersecção do tema da Blockchain com o contexto da Economia Compartilhada de forma a apresentar essa tecnologia como uma base de registros que pode auxiliar no desenvolvimento de serviços de compartiIhamento. Há também o estudo da Blockchain e sua eficácia com relação a questão de fraudes no âmbito das transações onli- ne, além do estudo dos motivos que levam o setor bancário estar focado na implantação de estratégias de Blockchain. Assim, as pesquisas dessas duas subáreas estão focadas em analisar como essa tecnologia afeta o negócios e contextos econômicos e tecnológicos. A seguir apresenta-se um quadro resumo (Quadro 3) dos perfis dos trabalhos publicados em Ciências Sociais e Aplicadas sobre Blockchain:

Tendo em vista esse panorama acerca do tema de Blockchain apresentam-se a seguir as considerações finais deste artigo.

\section{CONSIDERAÇÕES FINAIS}

O presente artigo alcançou o objetivo da pesquisa e respondeu ao questionamento proposto ao identificar e descrever o perfil das pesquisas realizadas sobre Blockchain em periódicos acadêmicos até 2017 (até o mês de abril de 2017). Sendo assim, ao realizar-se as análises foi possível identificar que os artigos publicados nesta te- 


\section{TABELA 5 - Principais resultados dos artigos da área de 'Accounting, Finance \& Economics' e 'Business, Management and Accounting}

\begin{tabular}{|c|c|c|}
\hline \multicolumn{3}{|c|}{ Área 'Accounting, Finance \& Economics' e 'Business, Management and Accounting' } \\
\hline Artigo & Objetivo & Resultados \\
\hline $\begin{array}{l}\text { A maturity model for } \\
\text { blockchain adoption }\end{array}$ & $\begin{array}{l}\text { Apresentar a taxonomia da avaliação da maturidade, que é } \\
\text { usada como Base para o desenvolvimento do Modelo de Ma- } \\
\text { turidade Blockchain (BCMM). Apresentar um procedimento } \\
\text { que facilita organizações em design e adoção de aplicativos } \\
\text { de cadeias de blocos. }\end{array}$ & $\begin{array}{l}\text { O modelo de maturidade Blockchain e seu processo de } \\
\text { adoção foram discutidos e apresentados de forma que este } \\
\text { estudo serve de guia às instituições para fazer decisões de } \\
\text { adoção de caderneta de forma mais sistemática. }\end{array}$ \\
\hline $\begin{array}{l}\text { Analysis and outlook of } \\
\text { applications of block- } \\
\text { chain technology to } \\
\text { equity crowdfunding in } \\
\text { China }\end{array}$ & $\begin{array}{l}\text { Examinar os problemas atuais na prática do crowdfunding de } \\
\text { capital na China com base na análise das características da } \\
\text { tecnologia Blockchain. Explorar ainda maias as aplicações } \\
\text { práticas dessas tecnologias em crowdfunding de capital. }\end{array}$ & $\begin{array}{l}\text { Do ponto de vista teórico, a tecnologia Blockchain é uma so- } \\
\text { lução que obtém registro de patrimônio eficiente e de baixo } \\
\text { custo, transação e transferência de capital e voto de acionistas } \\
\text { no setor de crowdfunding, eliminando os riscos legais relacio- } \\
\text { nados ao gerenciamento de fundos. Também pode ajudar os } \\
\text { reguladores a supervisionar e entender o mercado crowdfun- } \\
\text { ding. Assim, as aplicações de cadeias de blocos têm boas } \\
\text { perspectivas no domínio do crowdfunding de capital. }\end{array}$ \\
\hline $\begin{array}{l}\text { Blockchain application } \\
\text { and outlook in the } \\
\text { banking industry }\end{array}$ & $\begin{array}{l}\text { Explicitar por que o setor bancário atual está focado na } \\
\text { implantação de estratégias de Blockchain? Em quais cenários } \\
\text { específicos podem ser aplicadas a Blockchain? Quais são os } \\
\text { problemas existentes no processo de implementação dessa } \\
\text { tecnologia? }\end{array}$ & $\begin{array}{l}\text { A Blockchains poderia revolucionar a tecnologia subjacente } \\
\text { dos sistemas de compensação de pagamentos e de infor- } \\
\text { mações de crédito nos bancos, atualizando-os e transfor- } \\
\text { mando-os. As aplicações Blockchain também promovem a } \\
\text { formação de cenários "multicêntricos, fracamente interme- } \\
\text { diados", o que aumentará a eficiência do setor bancário. }\end{array}$ \\
\hline $\begin{array}{l}\text { Blockchain-based } \\
\text { sharing services: What } \\
\text { Blockchain technology } \\
\text { can contribute to smart } \\
\text { cities }\end{array}$ & $\begin{array}{l}\text { Propor quadro conceitual com três dimensões: humano, } \\
\text { tecnologia e organização, e explora um conjunto de fatores } \\
\text { fundamentais que tornam a cidade inteligente de uma econo- } \\
\text { mia compartilhada perspectiva. A partir desse modelo, busca } \\
\text { discutir as contribuições da Blockchain para esses fatores e } \\
\text { como seus elementos podem ajudar cidades inteligentes a } \\
\text { desenvolver serviços de compartilhamento. }\end{array}$ & $\begin{array}{l}\text { Framework que apresenta como os recursos da tecnologia } \\
\text { Blockchain podem contribuir para o desenvolvimento inteli- } \\
\text { gente da cidade através do compartilhamento de serviços. }\end{array}$ \\
\hline $\begin{array}{l}\text { Fraud detections for } \\
\text { online businesses: } \\
\text { a perspective from } \\
\text { Blockchain technology }\end{array}$ & $\begin{array}{l}\text { Explorar a fraude de classificação (rating) ao diferenciar a } \\
\text { fraude subjetiva da fraude objetiva. Em seguida, discutir a } \\
\text { eficácia da tecnologia Blockchain em fraude objetiva e sua } \\
\text { limitação na fraude subjetiva, especialmente a fraude de ra- } \\
\text { ting. Por fim, analisar sistematicamente a robustez dos siste- } \\
\text { mas de reputação baseados em cadeias de blocos em cada } \\
\text { tipo de fraude de rating no contexto de negócios online. }\end{array}$ & $\begin{array}{l}\text { A tecnologia Blockchain oferece novas oportunidades para re- } \\
\text { desenhar o sistema de reputação. Os sistemas Blockchain são } \\
\text { muito eficazes na prevenção de fraude de informações objeti- } \\
\text { vas, como a fraude de aplicativos de empréstimo, onde a infor- } \\
\text { mação fraudulenta é baseada em fato. No entanto, sua eficácia } \\
\text { é limitada em fraude de informação subjetiva, como a fraude de } \\
\text { rating, onde a verdade do solo não é facilmente validada. }\end{array}$ \\
\hline $\begin{array}{l}\text { Regulatory Issues in } \\
\text { Blockchain Technology }\end{array}$ & $\begin{array}{l}\text { Examinar os principais desafios regulatórios que afetam as } \\
\text { cadeias de blocos (Blockchain) na Europa e nos Estados } \\
\text { Unidos (EUA). }\end{array}$ & $\begin{array}{l}\text { A abordagem adotada na Europa e nos EUA é, em grande } \\
\text { medida, um bom presságio para as futuras contribuições } \\
\text { inovadoras da Blockchain, especialmente nos serviços fi- } \\
\text { nanceiros e setores relacionados. As descobertas fornecem } \\
\text { suporte para a tecnologia Blockchain avançar com freios } \\
\text { regulatórios mínimos para maior valor agregado e aprimo- } \\
\text { ramento de eficiência, especialmente para serviços finan- } \\
\text { ceiros, expandindo assim a inclusão financeira. }\end{array}$ \\
\hline $\begin{array}{l}\text { Trusting records: is } \\
\text { Blockchain technology } \\
\text { the answer? }\end{array}$ & $\begin{array}{l}\text { Explorar o valor da tecnologia Blockchain como uma solução } \\
\text { para criar e preservar registros digitais confiáveis, apresen- } \\
\text { tando algumas das limitações, riscos e oportunidades da } \\
\text { abordagem. }\end{array}$ & $\begin{array}{l}\text { A Blockchain pode ser usada para abordar problemas as- } \\
\text { sociados à integridade da informação, assumindo controles } \\
\text { de gerenciamento de infraestrutura e arquitetura de segu- } \\
\text { rança adequados. No entanto, não garante a confiabilidade } \\
\text { das informações em primeiro lugar e teria várias limitações } \\
\text { como uma solução a longo prazo para a manutenção de } \\
\text { registros digitais confiáveis. }\end{array}$ \\
\hline
\end{tabular}

Fonte: elaborado pelos autores (2019) 


\section{QUADRO 3 - Perfil dos Trabalhos Publicados em Ciências Sociaias Aplicadas sobre Blockchain}

Perfil dos Trabalhos Publicados em Ciências Sociais e Aplicadas sobre Blockchain

\section{Princiapais temas abordados:}

- regulamentação dessa tecnologia para um maior impacto e uso corporativo

- uso da tecnologia blockchain como uma base de registros que pode auxiliar no desenvolvimento de serviços de compartilhamento

(Economia Compartilhada)

- estudo da Blockchain e sua eficácia com relação a questão de fraudes no âmbito das transações online

- motivações do setor bancário para implantação de estratégias de Blockchain.

\section{Estudos futuros:}

Os trabalhos até então versam sobre o desenvolvimento tecnológico da Blockchain, assim como da possibilidade de adoção por alguns setores especificos (bancário, por exemplo) e contextos específicos (economia compartilhada, por exemplo), além disso há o desenvolvimento de discussões sobre a forma de regulamentação dessa tecnologia e proposições de modelo de maturidade e processo de adoção dessa tecnologia. Dessa forma, identificou-se que grande parte das discussões desenvolvidas nos estudos estão focadas em banefícios e desafios de possíveis cenários de adoção e uso dessa, havendo, portanto, a possibilidade do desenvolvimento de estudos de caso nesses tópicos em que já se iniciou o estudo e discussão teórica.

Fonte: elaborado pelos autores (2019)

mática são dos últimos 3 anos $(2015,2016$ e 2017), sendo que o ápice de publicações até o momento foi no ano de $2016 \mathrm{com} 20$ artigos dos 28 selecionados para análise de suas características.

Observou-se também que há uma pluralidade de abordagens dada a essa temáti$\mathrm{ca}$, tendo em vista a área de conhecimento dos periódicos em que foram publicados os artigos de forma a corroborar com as afirmações de autores em relação ao potencial disruptivo do Blockchain e sua não restrição de uso no Bitcoin (YERMACK, 2017; PILSINGYON, 20I6). Além disso, destaca-se que, tendo em vista os países de origem dos periódicos esse tema já possui espaço para pesquisa e debate em diversas localidades, havendo destaque para países como Estados Unidos, Países Baixos e Reino Unido.

Em relação aos temas abordados pelas pesquisas foi possível identificar uma variedade de possibilidades do uso da Blockchain, de forma que há diversas áreas do conhecimento estudando esse tema e não apenas áreas mais técnicas e que abordem a forma que a Blockchain deve estar estruturada. Nas análises realizadas consta- tou-se que há estudos que abordam temas como, por exemplo, regulação e possíveis aplicações em diversas áreas. Por fim destaca-se que as áreas de conhecimento dos 28 artigos analisados foram: Ciências Sociais Aplicadas, Ciências Exatas e da Terra, Engenharias, Ciência da Saúde e Linguística, Letras e Artes.

Por fim, como limitações dessa pesquisa tem-se a questão dos idiomas analisados em que, devido aos critérios de seleção dos artigos foi necessário excluir artigos em alemão, chinês e outros idiomas que não o português, inglês e espanhol. Além disso, como uma limitação, pode-se se destacar a não profundidade dada ao conteúdo de todos os artigos, sendo essa, portando um próximo aprofundamento a ser realizado. Ademais das questões já destacadas, pode-se sugerir para futuras pesquisas uma análise dos artigos publicados em eventos nacionais e internacionais sobre Blockchain de forma que seja possível comparar as abordagens dadas a esse conceito nos eventos com os resultados apresentados nesta pesquisa. Além disso, tendo em vista as análises realizadas sobre o perfil dos estudos sobre Blockchain, identificou-se 
a necessidade, pelos diversos campos do conhecimento, do desenvolvimento de estudos que analisem casos práticos da adoção dessa tecnologia e seus impactos nos negócios, tanto os impactos relacionados a estrutura tecnologica, quanto os impactos relacionados ao modelo de negócio e estrutura organizacional das empresas. 


\section{REFERÊNCIAS}

ATZORI, M. Blockchain Technology and Decentralized Governance: Is the State Still $\mathrm{Ne}$ cessary? New York: Social Science Research Network, 2015.

BARDIN, L. Análise de conteúdo. 70. ed. Lisboa: LDA, 20II.

GIL, A. C. Como elaborar projetos de pesquisa. 4. ed. São Paulo: Atlas, 2008.

LOTKA, A. J. The frequency distribution of scientific productivity. Journal of the Washington Academy of Sciences, v. 16, n. 12, p. 317-323, 1926.

MCKENDRICK, J. Why Blockchain May Be Your Next Supply Chain. 2017. Disponível em: https://www.forbes.com/sites/joemckendrick/20 I 7/04/2I/why-blockchain-may-be-your-next-supplychain/\#683f435cl3cf. Acesso em: 24/04/2017.

MUÑOZ, D. L. C. Estudos empíricos de gestão de conhecimento orientados para sustentabilidade: uma revisão sistemática de literatura de 1998 a 2009. 2009. 220 f. Dissertação (Programa de Pós-Graduação de Engenharia e Gestão de Conhecimento) - Universidade Federal de Santa Catarina (UFSC). 2009.
NAKAMOTO, S. Bitcoin: a peerto-peer electronic cash system. 2008. Disponivel em: http://bitcoin.org/bitcoin.pdf. Acesso em: 03/04/2017

NERY, C. Blockchain desafia grandes bancos. 2017. Disponível em: http://www2.valor.com.br/ empresas/4898008/blockchain-desafia-grandes-bancos. Acesso em: 24/04/20I7.

PILKINGTON, M. Blockchain Technology: Principles and Applications. In: OLLEROS, X.; ZHEGU, $M$ (Org.). Research Handbook on Digital Transformations. Massachusetts: Edward Elgar, 2016. cap. II.

ROUSSEAU, B.; ROUSSEAU, R. Percolation as a model for informetric distributions: fragment size distribution characterized by Bradford curves". Scientometrics, v. 47, p. 195-206, 2000.

STELER, F.W.; CERQUEIRA, A.H. Cinco princípios básicos do Blockchain e por que ele já está sendo chamado de a 'Internet dos Negócios’. 2017. Disponível em: http://cio.com. $\mathrm{br} /$ tecnologia/2017/03/06/cinco-principios-basicos-do-blockchain/.Acesso em: 24/04/20I7.
SWAN, M. Blockchain: blueprint for a New Economy. California: O'Reilly, 2015.

TAPSCOTT, D.; KIRKLAND, R. How blockchains could change the world. 2016 Disponivel em:http:// www.mckinsey.com/industries/ high-tech/our-insights/how-blockchains-could-change-the-world. Acesso em: 24/04/2017.

TAPSCOTT, D.; TAPSCOTT, A. Blockchain Revolution. New York: Penguin Random House LLC, 2016.

TAPSCOTT, D.; TAPSCOTT, A. How Blockchain Will Change Organizations. MITSloan Management Review. Winter 2017.

VALDES, R.; FURLONGER, D. Prepare for a multiple Blockchain world. 2016. Disponível em: https://www.forbes.com/ sites/gartnergroup/2016/07//5/ prepare-for-a-multiple-blockchain -world/\#26e7e0dd30ca. Acesso em: 24/04/2017.

WRIGHT, A.; DE FILIPPI, P. Decentralized Blockchain Technology and the Rise of Lex Cryptographia. New York: Social Science Research Network, 2015. YERMACK, D. Corporate Governance and Blockchains. Review of Finance. v. 2I, n. I, 2017. 
APÊNDICE A - LISTA DE ARTIGOS ANALISADOS

\begin{tabular}{|c|c|c|c|}
\hline TITULO & AUTORES & JOURNAL & ANO \\
\hline A maturity model for blockchain adoption & Huaiqing Wang; Kun Chen; Dongming Xu & Financial Innovation & 2016 \\
\hline $\begin{array}{l}\text { Analysis and outlook of applications of blockchain technology } \\
\text { to equity crowdfunding in China }\end{array}$ & Huasheng ZhuZach; Zhizhong Zhou & Financial Innovation & 2016 \\
\hline Behavior pattern clustering in blockchain networks & $\begin{array}{l}\text { Butian Huang; Zhenguang Liu; Jianhai } \\
\text { Chen; Anan Liu; Qi Liu; Qinming He }\end{array}$ & $\begin{array}{l}\text { Multimedia Tools and } \\
\text { Applications }\end{array}$ & 2017 \\
\hline Beyond Bitcoin: issues in regulating blockchain transactions & Trevor I. Kiviat & Duke Law Journal & 2015 \\
\hline $\begin{array}{l}\text { Bitcoin blockchain dynamics: The selfish-mine strategy in the } \\
\text { presence of propagation delay }\end{array}$ & $\begin{array}{l}\text { J. Göbel; H.P. Keeler; A.E. Krzesinski; P.G. } \\
\text { Taylor }\end{array}$ & Performance Evaluation & 2016 \\
\hline Blockchain & $\begin{array}{l}\text { Michael Nofer; Peter Gomber; Oliver Hinz; } \\
\text { Dirk Schiereck }\end{array}$ & $\begin{array}{l}\text { Business \& Information } \\
\text { Systems Engineering }\end{array}$ & 2017 \\
\hline Blockchain application and outlook in the banking industry & Ye Guo; Chen Liang & Financial Innovation & 2016 \\
\hline $\begin{array}{l}\text { Blockchain technology as a regulatory technology: From code } \\
\text { is law to law is code }\end{array}$ & P. De Filippi; S. Hassan & First Monday & 2016 \\
\hline $\begin{array}{l}\text { Blockchain technology in the chemical industry: Machine-to- } \\
\text { machine electricity market }\end{array}$ & $\begin{array}{l}\text { Janusz J. Sikorski; Joy Haughton; Markus } \\
\text { Kraft }\end{array}$ & Applied Energy & 2017 \\
\hline $\begin{array}{l}\text { Blockchain-based secure firmware update for embedded } \\
\text { devices in an Internet of Things environment }\end{array}$ & B. Lee; J.-H. Lee & Journal of Supercomputing & 2017 \\
\hline $\begin{array}{l}\text { Blockchain-based sharing services: What blockchain } \\
\text { technology can contribute to smart cities }\end{array}$ & Jianjun Sun; Jiaqi Yan; Kem Z. K. Zhang & Financial Innovation & 2016 \\
\hline $\begin{array}{l}\text { BlockVN: A Distributed Blockchain Based Vehicular Network } \\
\text { Architecture in Smart City }\end{array}$ & $\begin{array}{l}\text { Pradip Kumar Sharma; Seo Yeon Moon; } \\
\text { Jong Hyuk Park }\end{array}$ & $\begin{array}{l}\text { Journal of Indormation } \\
\text { Processing Systems }\end{array}$ & 2017 \\
\hline Difficulty control for blockchainbased consensus systems & Daniel Kraft & $\begin{array}{l}\text { Peer-to-peer Networking } \\
\text { and Applications }\end{array}$ & 2016 \\
\hline $\begin{array}{l}\text { Digital Art as 'Monetised Graphics': Enforcing Intellectual } \\
\text { Property on the Blockchain }\end{array}$ & Martin Zeilinger & Philosophy \& Technology & 2016 \\
\hline $\begin{array}{l}\text { FairAccess: a new Blockchain-based access control } \\
\text { framework for the Internet of Things }\end{array}$ & $\begin{array}{l}\text { A. Ouaddah; A. Abou Elkalam; A. Ait } \\
\text { Ouahman }\end{array}$ & $\begin{array}{l}\text { Security and Communication } \\
\text { Networks }\end{array}$ & 2016 \\
\hline $\begin{array}{l}\text { Fraud detections for online businesses: a perspective from } \\
\text { blockchain technology }\end{array}$ & Yuanfeng Cai; Dan Zhu & Financial Innovation & 2016 \\
\hline $\begin{array}{l}\text { Healthcare Data Gateways: Found Healthcare Intelligence on } \\
\text { Blockchain with Novel Privacy Risk Control }\end{array}$ & $\begin{array}{l}\text { Xiao Yue; Huiju Wang; Dawei Jin; } \\
\text { Mingqiang Li; Wei Jiang }\end{array}$ & Journal of Medical Systems & 2016 \\
\hline $\begin{array}{l}\text { Internet of Things, Blockchain and Shared Economy } \\
\text { Applications }\end{array}$ & $\begin{array}{l}\text { Steve Huckle; Rituparna Bhattacharya; } \\
\text { Martin White; Natalia Beloff }\end{array}$ & Procedia Computer Science & 2016 \\
\hline Regulatory Issues in Blockchain Technology & Peter Yeoh & $\begin{array}{l}\text { Journal of Financial } \\
\text { Regulation and Compliance }\end{array}$ & 2017 \\
\hline $\begin{array}{l}\text { Rep on the Roll: A Peer to Peer Reputation System } \\
\text { Based on a Rolling Blockchain }\end{array}$ & Richard Dennis; Gareth Owenson & $\begin{array}{l}\text { International Journal for } \\
\text { Digital Society }\end{array}$ & 2016 \\
\hline Socialism and the blockchain & S. Huckle; M. White & Future Internet & 2016 \\
\hline Story Blocks: Reimagining narrative through the blockchain & $\begin{array}{l}\text { Deborah Maxwell; Chris Speed; Larissa } \\
\text { Pschetz }\end{array}$ & Convergence & 2017 \\
\hline $\begin{array}{l}\text { The Blockchain as a Narrative Technology: Investigating } \\
\text { the Social Ontology and Normative Configurations of } \\
\text { Cryptocurrencies }\end{array}$ & Wessel Reijers; Mark Coeckelbergh & Philosophy \& Technology & 2016 \\
\hline $\begin{array}{l}\text { The loT electric business model: Using blockchain technology } \\
\text { for the internet of things }\end{array}$ & Yu Zhang; Jiangtao Wen & $\begin{array}{l}\text { Peer-to-Peer Networking } \\
\text { and Applications }\end{array}$ & 2016 \\
\hline Trusting records: is Blockchain technology the answer? & Victoria Louise Lemieux & $\begin{array}{l}\text { Records Management } \\
\text { Journal }\end{array}$ & 2016 \\
\hline $\begin{array}{l}\text { Two Technical Images: Blockchain and High-Frequency } \\
\text { Trading }\end{array}$ & Diego Viana & Philosophy \& Technology & 2016 \\
\hline $\begin{array}{l}\text { Walks trajectory tracking of shared information based on } \\
\text { consortium blockchain }\end{array}$ & J. Zhang & $\begin{array}{l}\text { Revista de la Facultad de } \\
\text { Ingenieria }\end{array}$ & 2016 \\
\hline $\begin{array}{l}\text { Where Is Current Research on Blockchain Technology?-A } \\
\text { Systematic Review. }\end{array}$ & $\begin{array}{l}\text { Jesse YliHuumo; Deokyoon Ko; Sujin Choi; } \\
\text { Sooyong Park; Kari Smolander }\end{array}$ & PLoS ONE & 2016 \\
\hline
\end{tabular}

Fonte: elaborado pelos autores (2018) 
APÊNDICE B - ÁREA E LOCAL DE ORIGEM DOS PERIÓDICOS

\begin{tabular}{|c|c|c|c|c|}
\hline JOURNAL & ÁREA & LOCAL & $\mathrm{H}$ index & $\begin{array}{c}\text { SJR } \\
(2015)\end{array}$ \\
\hline Applied Energy & Engineering & United Kingdom & 99 & 2,998 \\
\hline Business \& Information Systems Engineering & Computer Science & Germany & 22 & 0,63 \\
\hline Convergence & Arts and Humanities & United States & 25 & 0,844 \\
\hline Duke Law Journal & Social Sciences - Law & United States & 30 & 1,27 \\
\hline Financial Innovation & Accounting, Finance \& Economics & China & - & - \\
\hline First Monday & Computer Science & United States & 49 & 0,428 \\
\hline Future Internet & Internet technologies and the information society & Switzerland & - & - \\
\hline International Journal for Digital Society & Digital Society and Green Computing & United Kingdom & - & - \\
\hline Journal of Financial Regulation and Compliance & Accounting, Finance \& Economics & United Kingdom & - & - \\
\hline Journal of Information Processing Systems & Computer Science & South Korea & 13 & 0,218 \\
\hline Journal of Medical Systems & Computer Science; Health Professions & United States & 44 & 0,717 \\
\hline Journal of Supercomputing & Computer Science & Netherlands & 33 & 0,615 \\
\hline Multimedia Tools and Applications & Computer Science & Netherlands & 37 & 0,408 \\
\hline Peer-to-peer Networking and Applications & Computer Science & United States & 10 & 0,395 \\
\hline Performance Evaluation & Computer Science & Netherlands & 50 & 0,527 \\
\hline Philosophy \& Technology & Arts and Humanities & Netherlands & 8 & 0,311 \\
\hline PLoS ONE & Agricultural and Biological Sciences; Medicine & United States & 181 & 1,395 \\
\hline Procedia Computer Science & Computer Science & Netherlands & 21 & 0,314 \\
\hline Records Management Journal & Business, Management and Accounting & United Kingdom & 11 & 0,324 \\
\hline Revista de la Facultad de Ingenieria & Engineering & Colombia & 5 & 0,133 \\
\hline Security and Communication Networks & Computer Science & United States & 15 & 0,3 \\
\hline
\end{tabular}

Fonte: elaborado pelos autores (2018) 